\title{
Spiral coning manoeuvre for in-orbit low thrust characterisation in CubeSats
}

DOI:

10.1016/j.ast.2017.09.035

10.1016/j.ast.2017.09.035

\section{Document Version}

Accepted author manuscript

Link to publication record in Manchester Research Explorer

\section{Citation for published version (APA):}

Macario-Rojas, A., \& Smith, K. L. (2017). Spiral coning manoeuvre for in-orbit low thrust characterisation in CubeSats. Aerospace Science and Technology, 71. https://doi.org/10.1016/j.ast.2017.09.035, https://doi.org/10.1016/j.ast.2017.09.035

\section{Published in:}

Aerospace Science and Technology

\section{Citing this paper}

Please note that where the full-text provided on Manchester Research Explorer is the Author Accepted Manuscript or Proof version this may differ from the final Published version. If citing, it is advised that you check and use the publisher's definitive version.

\section{General rights}

Copyright and moral rights for the publications made accessible in the Research Explorer are retained by the authors and/or other copyright owners and it is a condition of accessing publications that users recognise and abide by the legal requirements associated with these rights.

\section{Takedown policy}

If you believe that this document breaches copyright please refer to the University of Manchester's Takedown Procedures [http://man.ac.uk/04Y6Bo] or contact uml.scholarlycommunications@manchester.ac.uk providing relevant details, so we can investigate your claim.

\section{OPEN ACCESS}




\title{
SPIRAL CONING MANOEUVRE FOR IN-ORBIT LOW THRUST CHARACTERISATION IN CUBESATS
}

\author{
A. Macario-Rojas ${ }^{\text {a,* }}$, K. L. Smith ${ }^{\mathrm{a}}$ \\ ${ }^{a}$ School of Mechanical Aerospace and Civil Engineering \\ The University of Manchester \\ M13 9PL, United Kingdom
}

\begin{abstract}
The ability to accurately measure the level of thrust during in-orbit operations is fundamental to the characterisation of emerging propulsion systems for nanosatellites. Many new CubeSat missions use propulsion systems with thrust levels in the order of few micro-Newtons. Whilst laboratory sensing resources are able to resolve such low thrust values, in complementary in-orbit characterisation are limited and in the main not compatible with the standard CubeSat mission. Additionally, typical in-orbit assessment of micro-thrust is generally carried out through body angular speed changes, the effectiveness of which is drastically reduced when external perturbations and sensor noise approach or exceed the thruster action on the CubeSat. This investigation sets out to improve in-orbit micro-thrust characterisation via changes in body angular velocity periodicity due to off-centred thrust action in nearly axisymmetric CubeSats. Unlike traditional methods that rely on determining angular acceleration this method employs a frequency analysis of the transversal component of the angular velocity signal with the aim of reducing measurement error. Numerical simulations support the feasibility and adequacy of the proposed low-thrust gauging method, particularly for weak and noisy sensor signals. The robustness of the method allows for interchangeable analysed signal and enables the use of simple commercial-off-the-shelf rate sensors in fine micro-thrust characterisation.
\end{abstract}

Keywords: CubeSat, Thrust characterisation, Manoeuvre

\section{Introduction}

A major breakthrough in the CubeSat standard development and applicability is the inclusion of functional propulsion systems that enable autonomous and controllable satellite dynamics. Adequate thrust characterisation is fundamental in the development, improvement, and optimization of propulsion technologies, however it is far from being a trivial task. In general, there exists a wide variety of space propulsion technologies developed to meet specific application requirements [16]. The selection of the propulsion system technology is often dictated by the delivered thrust level and working performance under the objective mission and environmental conditions. Thus, a method to correlate thrust level and input parameters, to identify performance dependancy on external factors with sufficient precision, is required for optimal employment of any kind of propulsion system. Thrust characterisation is an arduous task that commonly involves the identification of the thrust vector, beam or jet divergence, plume structure, transient and steady-state responses, vibration modes, thermal ranges, electrostatic charging, ambient effects, and supply dependencies amongst other factors. The role of relevant parameters involved in the thruster performance can be estimated by using

\footnotetext{
${ }^{*}$ Corresponding author

Email addresses: alejandromacario.rojas@manchester .ac .uk (A. Macario-Rojas), kate.smith@manchester.ac.uk (K. L. Smith)
}

direct thrust measurements or by assessing features associated to the thrust. However, whenever possible, direct thrust measurement is universally preferred $[17,21]$. Various characterisation methods have been devised in line with the thrust level of the systems. Whilst some aspects of high levels of thrust are easily measured, low thrust features are often barely noticeable even for high accuracy state-of-the-art measuring apparatuses. Thrust characterisation under laboratory conditions is commonly carried out using static test rigs, pendulum balances, torsion balances, and time-of-flight mass spectrometry. Additionally propulsion system characterisation tests for inspace operation are generally performed in vacuum environment adding complexity to the tests design, qualification, and implementation.

Reduced dynamic noise generation and fine throttle control capability in the range of $\mathrm{mN}$ to $\mu \mathrm{N}[5,38]$ are frequently considered to be of paramount importance in satellite propulsion system requirements. These considerations clearly reduce the margin of possible laboratory test conditions to a few constringent scenarios. In some cases, higher vacuum level requirements as well as propellant reactivity concerns add to the complexity of the characterisation [5]. For propulsion systems in the order of $\mu \mathrm{N}$, the characterisation can be particularly difficult because factors such as the weight of the thruster, stiffness of the feed and supply elements, ambient noise, thermal deformation, electrostatic and electromagnetic forces, and vacuum pump vibrations, are generally of higher magnitude than the thrust level 
itself overwhelming its action [5, 17, 42]. Another relevant aspect of these sort of laboratory tests is that in the absence of a dominant damping medium, primarily air, the start-up transient responses are long lasting. Despite all the above, current gauging technologies are able to resolve thrust values of few tens of $\mathrm{nN}$ for specific thruster characteristics [25]. However, important aspects of the ultimate operational environment (outer space) for satellite propulsion systems, have not been successfully reproduced in laboratory conditions. This makes supplementing laboratory data with in-orbit characterisation specially for low-thrust systems desirable [31].

CubeSats represent the current frontier of propulsion miniaturisation in space applications. Ongoing efforts are directed towards developing, testing, and characterising suitable systems for CubeSats. Current capabilities of nanosatellite propulsion systems range from tenths of $\mathrm{N}$ to few $\mu \mathrm{N}$ as illustrated in Fig. 1. Detailed information is reported in Table 2 in the appendix section.

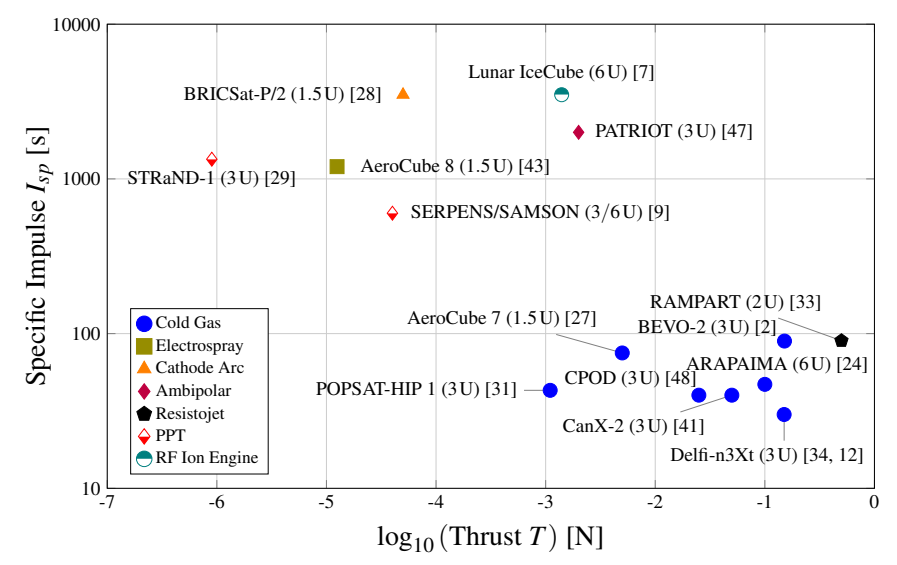

Figure 1: Log-log plot of reported propulsion systems in CubeSats for various size factors (inside the parentheses) thus far. The value of $I_{s p}$ is proportional to the efficiency of propellant mass consumption in the production of thrust for a given propulsion system. This investigation focuses on $T$ values around $\mu \mathrm{N}$ encompassing current high $I_{s p}$ engines and the throttle range of other thruster technologies.

Although propulsion system operational characteristics may be varied amongst technologies, general in-orbit thrust characterisation in CubeSats relies upon attitude changes and the resolution of onboard attitude determination resources. Acceptable attitude determination is of central importance for system characterisation through in-orbit attitude manoeuvres such as detumbling, target pointing (Sun, Nadir, etc.), and controlled spin $[13,31,34]$. Ongoing efforts of space industry and private research are directed towards the development and adaptation of suitable sensing technologies to the reduced space and power resources of CubeSats.

A survey of available attitude determination approaches in CubeSats from randomly selected historical nanosatellite missions is shown Fig. 2. For the sake of homogeneity, the selected nanosatellites are approximated to standard CubeSat form factors (when necessary) and sensors with similar characteristics are grouped into eight representative classifications. Fine and coarse sensors measuring the Sun vector (2-axis) may include specialised or Commercial Off-The-Shelf (COTS) elements. Some missions like the surveyed Colony- 1 and GeneSat-1, use solar panels power input for coarse solar vector estimations. The second group includes missions using the stars as reference frame (2-axis). This group encompasses high-end missions like the RAVAN, MinXSS, Aalto-1, STARE, AeroCube 7-OCSD, CADRE, and SENSE. The third group uses the Earth shape as reference (2-axis) mostly in the form of horizon sensors (in configurations of COTS IR pyroelectric sensors). This group include missions like the DICE, Drag-free CubeSat, GOMX-3, AeroCube-3, AeroCube-4, CUTE-1.7+APD-2, and AeroCube 7-OCSD. The Earth's magnetic field is amply used for 3-axis attitude determination in all nanosatellite size factors as shown in the fourth group in Fig. 2. In this case, magnetometers mostly in the form of COTS elements, provide local magnetic field measurements that are compared to onboard reference Earth magnetic field models, e.g. the International Geomagnetic Reference Field (IGRF) or the World Magnetic Model (WMM), to estimate spacecraft attitude. Although the space environment produces fluctuations in the Earth's magnetic field, which increase with altitude, available models capture most of its characteristics in low LEO enabling reliable coarse estimations. CubeSats have greatly benefitted from the development, optimisation, and miniaturisation of Global Navigation Satellite System (GNSS) receivers from the electronics consumer market. COTS GNSS receivers assists enhanced attitude estimations by providing superior spatial identification to complement star map and Earth magnetic models. Although in some cases location continues to be estimated by onboard orbit propagations, the use of GNSS receivers is gradually implemented in all CubeSat form factors. In a similar way to GNSS technologies, MicroElectroMechanical Systems (MEMS) are in constant improvement to meet the electronics consumer market demand. The basic Inertial Measurement Unit (IMU) in standard CubeSats is composed of COTS MEMS accelerometers and gyroscopes providing a simple and low-cost solution for attitude variation identification. However, intrinsic fluctuations within the fundamental device operation gradually add measurement errors, namely bias or drift instability, which need correction. Eventual device calibration, i.e. zeroing, is normally enough to mitigate this fact. In spite of this, MEMS gyroscopes provide the current best short-term reference for 3-axis attitude determination in CubeSats. In practice each group shown in Fig. 2 may contribute to form an integral estimation of spacecraft attitude. Raw data from a variety of different sensors can be combined, e.g. using Kalman filtering, to furnish improved attitude estimations.

For CubeSat missions, it is therefore highly desirable to devise methods capable of drawing upon simple sensing resources to support effective thruster characterisation. The purpose of this investigation is to provide an alternative and effective method to resolve low thrust levels with COTS sensors for in-orbit low thrust characterisation in standard CubeSat configurations. Although this investigation encompasses $1.5 \mathrm{U}$ and $3 \mathrm{U}$ CubeSat form factors, the proposed methodology can be applied to other nearly symmetric geometries wherein appendages do not represent an important source of short term 


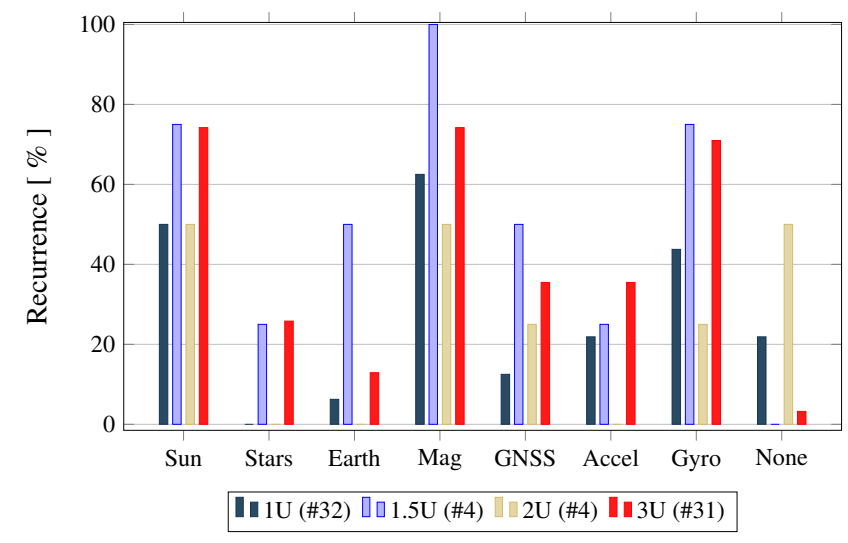

Figure 2: Resources for attitude determination in CubeSats. Data from a random sample of CubeSats missions. The surveyed number of each size factor is reported in parentheses.

attitude changes as discussed below.

Section 2 presents the general assumptions and mathematical description of the proposed methodology. Finally, Section 3 presents computer-simulated thrust characterisation examples aiming the comparison of the proposed method to a standard one for the evaluation of relative performance.

\section{The proposed method}

Acknowledging the predominant CubeSat size factors presented in Fig. 1, this investigation focuses on standard 1.5U and $3 \mathrm{U}$ CubeSats and thrust values ranging from $50 \mathrm{nN}$ to $10 \mu \mathrm{N}$. This range encompasses high performance propulsion systems with the lowest thrust levels reported in Fig. 1, and typifies a range difficult to characterise with standard attitude determination systems in CubeSats. The CubeSat geometries in this investigation are assumed as standard with near-symmetry in two-axis, namely axisymmetric, and the effect of appendages over the attitude evolution during the thrust characterisation considered negligible. Centroid and moment of inertia tensor variations are also assumed negligible. In this way, the CubeSat models employed in this investigation are rectangular parallelepipeds with the $z$ body axis parallel to the principal moment of inertia $I_{z}$ (minor axis of inertia), and the values of $I_{x}$ and $I_{y}$ nearly equivalent as shown in Fig. 3. These assumptions are in close compliance with reported CubeSat configurations $[13,27,41]$ and principal moments of inertia values in actual missions [3, 32, 40]. Additionally, this investigation is based on the common practice of performing in-orbit thruster characterisation via attitude changes $[13,27,29]$. To this end, a four-element thruster set-up configuration is used in the simulations due to the versatile and convenient possible spectrum of manoeuvres.

\subsection{System dynamics key concepts}

In order to provide a qualitative description of the method the following general concepts are considered. The angular momentum, $\boldsymbol{L}$, of a rigid body in torque-free motion remains fixed

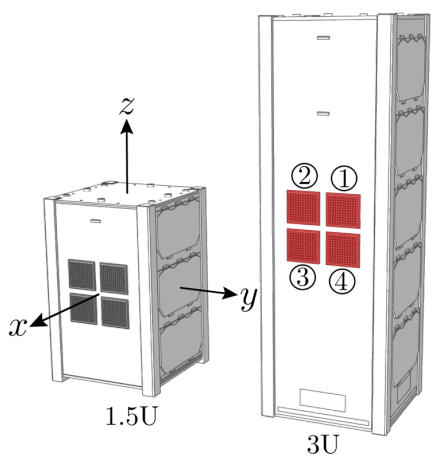

Figure 3: Reference CubeSats, body axes, and thruster patch units definition. The selected generic multi-thruster configuration for this investigation, provides rich manoeuvring possibilities. An instance of this is found in the ongoing BRICSat-P mission [13].

in inertial space and its magnitude does not vary with time. Two constants of the motion about the origin are then, the angular momentum and the kinetic energy, $K$. These constants are computed with the dyadic product in Eq. (1), and the dot product in Eq. (2) respectively, wherein $\boldsymbol{\omega}$ is the angular velocity vector in body axes and $\boldsymbol{I}$ is the inertia tensor.

$$
\begin{gathered}
\boldsymbol{L}=\boldsymbol{I} \boldsymbol{\omega} \\
K=\frac{\boldsymbol{\omega} \cdot \boldsymbol{L}}{2}
\end{gathered}
$$

Let $\boldsymbol{n}$ be a unit normal vector parallel to $\boldsymbol{\omega}$, so that $\boldsymbol{\omega}=\boldsymbol{\omega n}$. The moment of inertia about the axis of rotation $\boldsymbol{n}$ in principal axes (diagonal inertia tensor) is defined by

$$
\boldsymbol{I}=\boldsymbol{n} \cdot \mathbf{I n}
$$

Here, the Poinsot's construction provides a useful geometrical description of the rigid body motion by allowing the visualisation of $\boldsymbol{\omega}$ in terms of the constants of the motion. Consider the convenient vector description given by Eq. (4) [20] for the Poinsot's construction in which the direction of $\boldsymbol{\rho}$ is defined by $\boldsymbol{\omega}$ and its magnitude by the relative inertial properties of the body.

$$
\rho=\frac{n}{\sqrt{I}}=\frac{\omega}{\sqrt{2 K}}
$$

Assuming the definition in Eq. (5) leads to a function that has the form of a triaxial ellipsoid in cartesian $\boldsymbol{\rho}$ coordinates for constant values of $f$. It is of interest for the intended explanation, the particular case wherein $f(\rho)=1$, known as inertial ellipsoid.

$$
f(\rho)=\boldsymbol{\rho} \cdot \boldsymbol{I} \boldsymbol{\rho}=I_{x} \rho_{x}^{2}+I_{y} \rho_{y}^{2}+I_{z} \rho_{z}^{2}
$$

The gradient of function Eq. (5), furnishes information about the normal vectors of the inertial ellipsoid surface.

$$
\nabla_{\rho} f=2 \boldsymbol{I} \cdot \boldsymbol{\rho}=\sqrt{\frac{2}{K}} \boldsymbol{L}
$$


A relevant aspect of the motion arises from Eq. (6) that the surface normals of the inertial ellipsoid are parallel to the constant vector $\boldsymbol{L}$. Furthermore, by considering the projection, $h$, of the time dependent $\boldsymbol{\rho}$ on $\boldsymbol{L}$ in Eq. (7) it becomes evident that $h$ remains constant as it is defined solely by constants of the motion.

$$
h=\frac{\boldsymbol{\rho} \cdot \boldsymbol{L}}{L}=\frac{\sqrt{2 K}}{L}
$$

By joining and applying the aforementioned inferences to a tumbling regular prolate cuboid $\left(I_{x} \approx I_{y}>I_{z}\right)$ it is possible to illustrate the state of $\boldsymbol{\omega}$ as shown in Fig. 4. The construction of the illustration in inertial space can be realised by first defining the invariant $\boldsymbol{L}$. The vector $\boldsymbol{L}$ starts at the cuboid's centre of mass, and has been chosen pointing downwards in Fig. 4 for illustrative purposes. Following with the construction of other geometry elements around this vector, we know from Eq. (7) that there exists a fixed distance $h$ from the centre of mass to the projection of $\boldsymbol{\rho}$ on $\boldsymbol{L}$ at any time. Furthermore, Eq. (6) establishes that the inertial ellipsoid has to have its instantaneous surface normal, parallel to $\boldsymbol{L}$ for any $\boldsymbol{\rho}$. These two conditions are fulfilled by a plane at a fixed distance $h$ from the centre of mass of the body, named the invariable plane, over which the inertial ellipsoid rotates to preserve the constant relationship of $\boldsymbol{L}$ and $\boldsymbol{\rho}$ for any time. In this way, the instantaneous contact point between the inertial ellipsoid and the invariable plane in intermediate axis spinners trace out two circles, the polhode and herpolhode respectively. Finally, note that with respect to the body axes, $\boldsymbol{\rho}$ sweeps out a cone, referred to as the body cone. Likewise, the swept out cone in inertial space is called the space cone. Both idealised cones are always in contact, rolling one over the other through the contact line defined by $\boldsymbol{\rho}$.

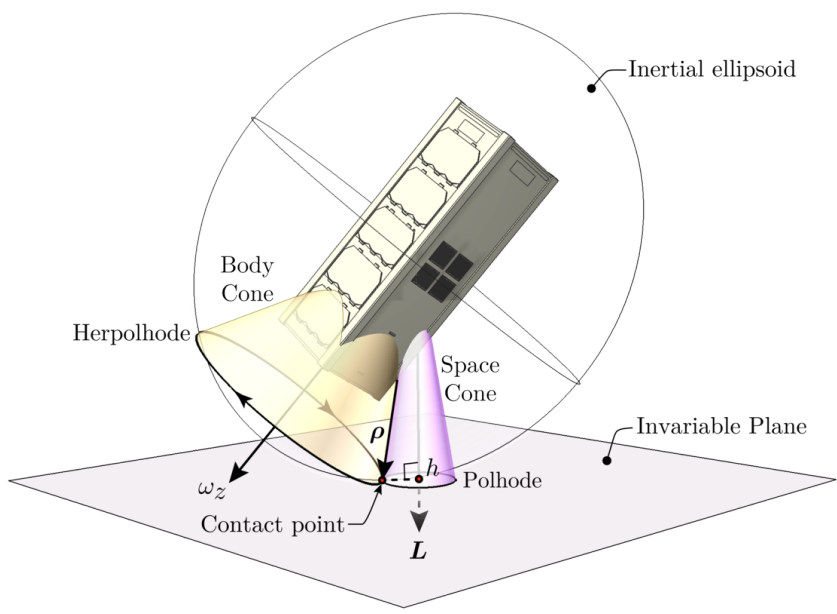

Figure 4: Poinsot's construction. The 3U CubeSat geometry has been included for illustrative purposes to emphasise the precession of $\boldsymbol{\rho}$ in body axes for a intermediate axis spinner. The herpolhode sweep-out frequency outlines the proposed methodology for in-orbit low thrust characterisation in CubeSats.

For the purpose of this investigation, the interpretation of Fig. 4 in body axes, wherein $\boldsymbol{\rho}$ has prograde precession around $\omega_{z}$ in an intermediate axis spinner is of relevance. Bearing in mind that $\boldsymbol{\rho}$ and $\boldsymbol{\omega}$ are collinear, this investigation employs the pre and post manoeuvre body cones of $\boldsymbol{\omega}$ to draw information about the thrust magnitude. The two cones can be idealised as two concentric nested cones with their apices located at the CubeSat's centre of mass and having different heights. In this particular case, concentricity in the body cones implies that the values of $\omega_{x}$ and $\omega_{y}$ remain constant during the test manoeuvre that aims change in $\omega_{z}$, which in turn modifies the inertial ellipsoid size. A distinctive open spiral cone, not used in the following descriptions, transitions from the pre to the post manoeuvre body cones. The conventional way to assess thrust action using body angular velocities is by observing the rate of change in body cone height, or put in another way, by assessing the rate of change in $\omega_{z}$ during the thrust manoeuvre. Provided that the thrust magnitude and sensing resources allow it, this method gives a simple and reliable method to evaluate thrust. Conversely, this investigation proposes the use of sweep-out frequency change of the the herpolhode, identifiable from $\omega_{x}$ or $\omega_{y}$, to draw information about the thrust magnitude. In principle, if the height difference of the body cones (or rate of change) is difficult to resolve, e.g. noisy signal and weak angular rates, it is still possible to identify changes in their characteristic and definable transversal ( $x y$ plane) sweep-out frequencies. Note that there exists redundancy in the data source of the sweepout frequency ${ }^{1}$ conferring additional flexibility to the proposed method.

Another important aspect to consider in the proposed method is that the cone angles of aperture are dictated by the values of $\boldsymbol{I}$ and the magnitude of the transversal component of $\boldsymbol{\omega}$, henceforth referred to as $\omega_{t}$. Two relevant cases are formed when the inertial ellipsoid becomes a sphere for $I_{x} \approx I_{y} \approx I_{z}$, and when $\omega_{t}=0$. In both cases $\boldsymbol{\rho}$ and $\boldsymbol{L}$ become collinear implying the disappearance of the space cone. The first case nullifies the sweep-out frequency of interest by establishing constant values of $\omega_{x}$ or $\omega_{y}$. Further discussion on the matter is presented in Section 2.2. At this point it is worth mentioning that the parallelism between $\boldsymbol{\rho}$ and $\boldsymbol{L}$ implies stable rotation under kinetic energy dissipative processes. However only unstable rotation in a prolate body (intermediate axis spinner) is of interest for this investigation as the analysis is based on the body cone sweepout frequency.

The effects of spacecraft mass variations and unstable rotation under energy dissipative phenomena, e.g. flexible elements such as antennae, become important over attitude evolution normally after long periods of time in comparison to the average thrust test. These topics are addressed in subsections 2.3 and 2.4 after the derivation of the pertinent analytical solutions of the rigid body motion in subsection 2.2.

\subsection{Analytic Solutions}

In order to develop the analytical framework of this investigation, consider Euler's equations of motion of a rotating rigid body with principal axes at the centre of mass. Perturbing

${ }^{1}$ Both $\omega_{x}$ and $\omega_{y}$ equally define the sweep-out herpolhode frequency. 
torques acting on the body motion are represented by the vector M.

$$
\begin{aligned}
M_{x} & =I_{x} \dot{\omega}_{x}+\left(I_{z}-I_{y}\right) \omega_{y} \omega_{z} \\
M_{y} & =I_{y} \dot{\omega}_{y}+\left(I_{x}-I_{z}\right) \omega_{z} \omega_{x} \\
M_{z} & =I_{z} \dot{\omega}_{z}+\left(I_{y}-I_{x}\right) \omega_{x} \omega_{y}
\end{aligned}
$$

These equations are a set of coupled nonlinear equations describing the temporal evolution of the angular velocity in body axes. Their general solution requires numeric integration, but in specific cases it is possible to obtain analytical solutions. One such case is for an axisymmetric body. Assuming that $I_{x}$ is nearly equivalent to $I_{y}$, Eq. (10) is simplified to Eq. (11) prompting decoupling and the solution of the system of equations.

$$
\omega_{z}=\frac{M_{z}}{I_{z}} t+\omega_{z_{0}}
$$

The simplest solution, excluding the trivial one, is found when the values of $M_{x}$ or $M_{y}$, and $M_{z}$ are negligible. This case describes a slender rigid body in forced rotation about its minimum axis of inertia. However, for the purpose of this investigation $M_{z} \neq 0$, i.e. in the characterising manoeuvre the thrust modifies $M_{z}$. Under this scenario, finding analytical solutions requires a far more complex approach. This investigation uses the methodology proposed in the work of Tsiotras and Longuski [46] to find a suitable solution for the problem at hand.

Eq. (12) presents the differential equation in complex notation for the transversal angular velocities. The variables in this equation are defined by Eqs. (13) to (18) with $A \equiv \omega_{z}$.

$$
\begin{aligned}
F=\dot{\mathbf{\Omega}}+i D A \boldsymbol{\Omega} \\
\mathbf{\Omega} \equiv \omega_{1} \sqrt{C_{2}}+i \omega_{2} \sqrt{C_{1}} \\
F \equiv \frac{M_{x}}{I_{x}} \frac{I_{z}}{M_{z}} \sqrt{C_{2}}+i \frac{M_{y}}{I_{y}} \frac{I_{z}}{M_{z}} \sqrt{C_{1}} \\
C_{1} \equiv \frac{I_{z}-I_{y}}{I_{x}} \\
C_{2} \equiv \frac{I_{z}-I_{x}}{I_{y}} \\
C \equiv \sqrt{C_{1} C_{2}} \\
D \equiv C \frac{I_{z}}{M_{z}}
\end{aligned}
$$

The integral of Eq. (12) with respect to $A$ and solved for $\boldsymbol{\Omega}$ represents the solution for the transversal angular velocities given by Eq. (19)

$$
\boldsymbol{\Omega}=\boldsymbol{\Omega}_{0} e^{i \frac{D}{2}\left(A^{2}-A_{0}^{2}\right)}+F e^{i \frac{D A^{2}}{2}} \int_{A_{0}}^{A} e^{-i \frac{D u^{2}}{2}} d u
$$

By neglecting $M_{x}, M_{y}$, and substituting $\boldsymbol{\Omega}_{0}=\omega_{x_{0}} \sqrt{C_{2}}+$ $i \omega_{y_{0}} \sqrt{C_{1}}, A_{0} \equiv \omega_{z_{0}}$, and $I_{x}$ and $I_{y}$ with $I_{t}$ for simplification purposes, yields Eq. (20)

$$
\boldsymbol{\Omega}=\left(\omega_{x_{0}}+i \omega_{y_{0}}\right) e^{i \frac{\left(I_{t}-I_{z}\right)\left(M_{z} t^{2}+2 I_{z} \omega_{z_{0}} t\right)}{2 I_{z} I_{t}}}
$$

Or equivalently in trigonometric form

$$
\boldsymbol{\Omega}=\left(\omega_{x_{0}}+i \omega_{y_{0}}\right)(\cos w t+i \sin w t)
$$

in which the angular frequency, $w$, is given by Eq. (22). Notice the explicit dependence of this spinning axisymmetric forced frequency on time in a characteristic linear chirp signal.

$$
w=\frac{\left(I_{t}-I_{z}\right)\left(M_{z} t+2 I_{z} \omega_{z_{0}}\right)}{2 I_{z} I_{t}}
$$

The real and imaginary components in Eq. (21) constitute the transversal angular velocities $\omega_{x}$ and $\omega_{y}$ respectively

$$
\begin{aligned}
& \omega_{x}=\frac{\mathfrak{R}(\boldsymbol{\Omega})}{\sqrt{C_{2}}} \\
& \omega_{y}=\frac{\mathfrak{I}(\boldsymbol{\Omega})}{\sqrt{C_{1}}}
\end{aligned}
$$

Finally, the rigid body angular velocities for $M_{x}=M_{y}=0$, and $M_{z} \neq 0$ are

$$
\begin{aligned}
& \omega_{x}=\omega_{x_{0}} \cos w t+\omega_{y_{0}} \sin w t \\
& \omega_{y}=\omega_{y_{0}} \cos w t-\omega_{x_{0}} \sin w t \\
& \omega_{z}=\frac{M_{z}}{I_{z}} t+\omega_{z_{0}}
\end{aligned}
$$

Furthermore, the free spin condition is fulfilled for $M_{z}=0$. In this case Eq. (25) to Eq. (27) are simplified to

$$
\begin{aligned}
& \omega_{x}=\omega_{x_{0}} \cos w_{n} t+\omega_{y_{0}} \sin w_{n} t \\
& \omega_{y}=\omega_{y_{0}} \cos w_{n} t-\omega_{x_{0}} \sin w_{n} t \\
& \omega_{z}=\omega_{z_{0}}
\end{aligned}
$$

with the spinning axisymmetric natural frequency defined by

$$
w_{n}=\omega_{z_{0}}\left(1-\frac{I_{z}}{I_{t}}\right)
$$

As a final remark in this subsection note that the projection of $\boldsymbol{\omega}$ on the $x y$ plane in body axes draw circles representing the herpolhode for both the free and forced spin conditions. In addition, it has been discussed in subsection 2 that for $I_{x} \approx I_{y} \approx I_{z}$ in torque-free motion, the body angular velocities are constant. This argument is now validated by Eq. (31) by providing a null transversal natural frequency for full symmetry conditions.

\subsection{Induced error due to temporal mass variation}

Temporal changes in mass quantity and distribution, e.g. propellant consumption, can be incorporated in the proposed method. However, it is convenient to recall that this investigation assumes $I_{t} \equiv I_{x} \approx I_{y}$. If important variation within the transversal component exists or is expected to occur implying necessary asymmetry, then bespoke analytical solutions may be still available [46], enabling thrust estimation with the proposed method. Fig. 5 presents the percent relative error of Eq. (22) to variations in $I_{t}$ and $I_{z}$ for standard 1.5U and 3U CubeSats, and the empirical value $w=0.01 \mathrm{rads}^{-1}$ (distinctive for $10 \mathrm{~min}$ of 
micro-thrust in a $1.5 \mathrm{U}$ CubeSat). The exhaust mass flow rate is computed with $\dot{m}_{e}=M_{z} / d_{m} I_{s p} g_{0}$ [11, pp. 557], being $d_{m}$ the effective moment arm reasonably equivalent to one fourth of the CubeSat face width accommodating a symmetric thruster array, namely $2.5 \mathrm{~cm}$, and $g_{0}$, the standard surface gravity. The value of $\chi$ is the product of $I_{s p}$ and $d_{m}$. The value of $I_{s p}$ according to Fig. 1, is typically higher than 1000 s for propulsion systems natively below $\mu \mathrm{N}$ yielding $\chi>25$. Under these assumptions, the induced error due to neglecting temporal mass variations is lower than ten thousandths percent.

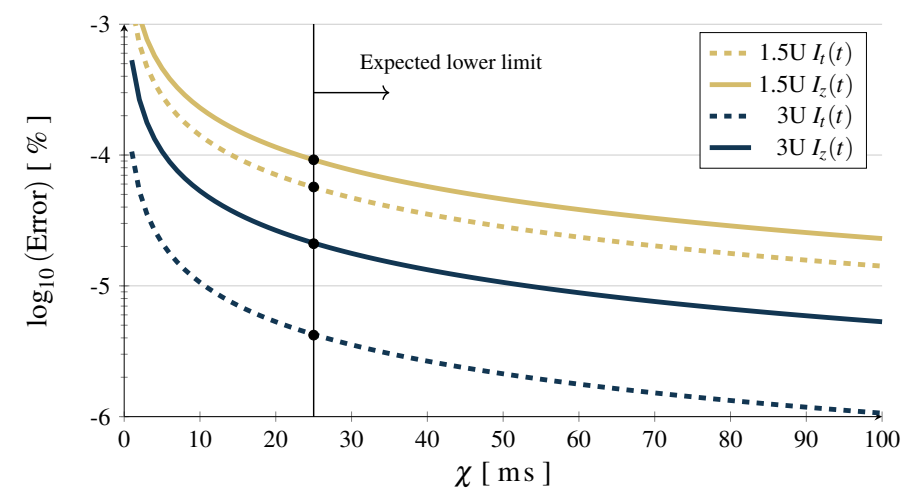

Figure 5: Log-lin plot of the percent relative error of the method due to transversal, $I_{t}(t)$, and longitudinal, $I_{z}(t)$, moment of inertia variations. The figure accounts for the propellant mass consumption led by $I_{s p}$ through $\chi$, the product of $I_{s p}$ and the moment arm, in standard CubeSat form factors.

\subsection{Rotation stability and energy dissipation}

Stable rotation in a simple axisymmetric spinner occurs around two preferred orthogonal axes satisfying parallelism between $\boldsymbol{\rho}$ and $\boldsymbol{L}$ as discussed before. Under non-conservative circumstances, an intermediate axis spinner, as in Fig. 4, would nutate until it spins about the major axis of inertia and minimum energy state. An explanation can be formulated by considering the constants of the motion and the moment of inertia as seen by $\boldsymbol{\omega}$. Given the attitude rotation energy by $K=1 / 2 I \omega^{2}$ and the angular momentum magnitude by $L=I \omega$, a torque-free spinner is then governed by $I=L^{2} / 2 K$. Energy loss mechanisms lower the value of $K$ giving rise to an increment in $I$. In this way the spinner would gradually move to stable rotation about the maximum moment of inertia (flat spin).

The source of predominant energy dissipation varies significantly amongst satellite designs. However, rotational power loss $\dot{K}$ is commonly related to hysteretic damping, for instance in flexible elements such as antennae booms being periodically bent by the action of the gravity-gradient field or in propellant slosh. The interaction of the Earth's magnetic field with metallic structural elements, i.e. eddy-current damping, is apparently a highly relevant factor in rotational energy dissipation in CubeSats $[26,36]$. As a dimensioning example on this point, consider that for an aluminium $-7075^{2}$ outer shell in a $3 \mathrm{U}$ Cube-

\footnotetext{
${ }^{2}$ Material in compliance with the CubeSat Design Specification [8], CubeSat configuration assumed in this investigation, $\omega_{z}=10 \%$, magnetic field acting parallel to the transversal plane and magnitude equivalent to the average at $100 \mathrm{~km}$ Earth altitude, and formulae from Barranger [4].
}

Sat immersed in a fluctuating magnetic field with flux density of $30 \mu \mathrm{T}$, the magnitude of eddy-current power loss is approximately 27 orders of magnitude lower than the input rotational power due to $1 \mu \mathrm{N}$ thrust.

$$
\frac{d \omega_{t}^{2}}{d t}=2 \frac{I_{z}}{I_{t}} \frac{\dot{K}}{I_{z}-I_{t}}
$$

Eq. (32) [11, pp. 489] describes the temporal evolution of $\omega_{t}$ due to energy dissipation in a prolate spinner. Although the sensitivity in $\omega_{t}$ due to energy dissipation is higher as $I_{t} \rightarrow I_{z}$, as shown in the plot of Eq. (32) in Fig. 6, it is safe to neglect its effects during the time span for the proposed thrust characterisation method without any loss of generality. Nonetheless, the proposed method may not be applicable to CubeSats with abnormal sources of attitude uncertainty like passive magnetic attitude control systems.

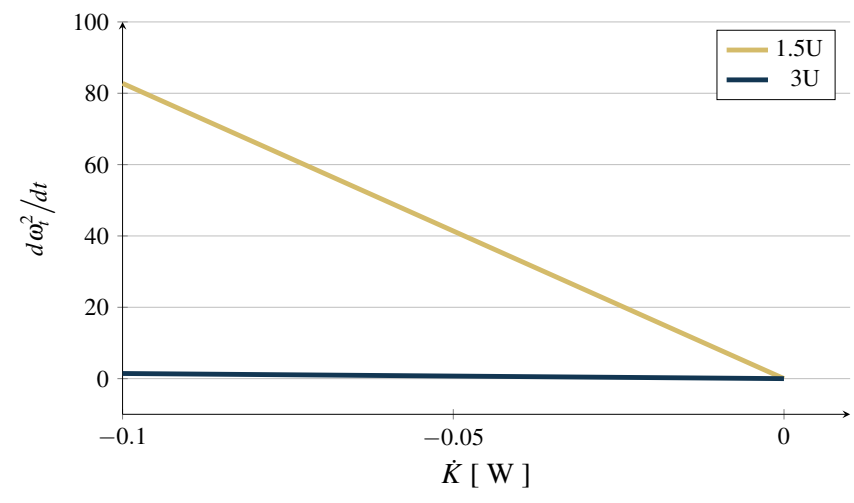

Figure 6: Effect of dissipative processes over the transversal angular velocities evolution in standard CubeSats. Values different from zero in $d \omega_{t}^{2} / d t$ amount to instability in a prolate spinner. The value of $\dot{K}$ is negative in power loss. 3U CubeSats are less prone to $\omega_{t}$ variation due to rotational power loss.

\section{Application example}

In order to estimate the desired thrust value, from the quotient of $M_{z}$ and the moment arm, it is necessary to solve Eq. (22) for $M_{z}$. Eq. (33) describes the temporal evolution of $M_{z}$ as a function of the spinning forced frequency $w$ and the initial angular velocity $\omega_{z_{0}}$ during the manoeuvre.

$$
M_{z}=\frac{I_{t} I_{z}}{\left(I_{t}-I_{z}\right) t} w-\frac{I_{z} \omega_{z_{0}}}{t}
$$

Typically spin-despin manoeuvres are used to estimate thrust levels through $M_{z}$ by assessing the imposed angular acceleration in one axis, e.g. assuming $I_{x} \approx I_{y}$ then simply $M_{z}=I_{z} \dot{\omega}_{z}$. On the other hand, estimations using Eq. (33) are dependent upon $\omega_{z}$ and $w$. By considering actual electronic angular rate sensors, both methods are affected by inherent signal noise and satisfactory definition of the magnitude of $\omega$. Moreover, thrust estimations from noisy signals using Eq. (33) can be poorer than those from the typical method (from $\dot{\omega}_{z}$ ) due to the additional dependence on $w$. The convenience of using Eq. (33) is evident when the analysis is centred in the pre/post manoeuvre transversal angular velocity responses, because transversal 
spinning axisymmetric natural frequencies can be determined with greater efficacy than magnitudes in signals difficult to resolve. In order to eliminate the dependency of Eq. (33) on $\omega_{z_{0}}$, we can use Eq. (22) to obtain the difference of the pre/post transversal natural frequencies, $\Delta w$, yielding the alternative Eq. (34).

$$
M_{z}=\frac{I_{t} I_{z}}{I_{t}-I_{z}} \frac{\Delta w}{t}
$$

In this way, the proposed method provides a suitable approach using standard attitude sensor resources in CubeSats for the characterisation of weak thrust levels otherwise strongly linked to sensor noise.

Simulated digital MEMS Gyro signals in three-axis are used in the following example. Basically, the sensor model comprises a second order transfer function with band-limited white noise wherein the noise power is defined by the Rate Noise Density reported in Table 1 . The simulation parameters aim to describe a distinctive low Earth orbit insertion opportunity for the typical CubeSat mission. The propagations use perturbation forces from gravitation, third-body, solar radiation pressure, atmospheric drag, and gravity gradient, acting on 3D models of the $1.5 \mathrm{U}$ and $3 \mathrm{U}$ CubeSats. Additionally, the cartesian coordinate axes coincide with the mutually orthogonal principal axes of rotation (principal moments of inertia), as assumed along this investigation.

In actual circumstances the proposed method for in-orbit thrust characterisation might encompass two manoeuvres. Fig. 7 and Video 1 (online version only) present the sequence of key events during the proposed thrust characterisation. A preliminary manoeuvre, referred to as Manoeuvre 1 in the figure, would bring the periodic transversal component of the angular velocity vector into the range of the sensing capabilities of the on-board attitude determination system resources in order to determine the axisymmetric natural frequency during a first coast period. As the torque level required for this first manoeuvre depends on specific mission conditions and constraints, such as signal noise level and initial body angular rates, this investigation focuses only on the second manoeuvre (Manoeuvre 2) aimed to characterise the thrust level. However, typical after deployment tumbling rates in CubeSats may range over a few degrees per second providing an adequate baseline natural frequency during the initial coasting period to dispense with a first manoeuvre. In this example we use the arbitrary but representative $[22,50]$ after deployment values of $\omega_{x_{0}}=1.1 \%$, $\omega_{y_{0}}=1.3 \%$, and $\omega_{z_{0}}=1.6 \%$ s. These initial rate values in conjunction with the sensor capabilities used in this example, allow us to dispense with the first manoeuvre.

Of interest for the proposed method are the plots of $\omega_{x}$ and $\omega_{y}$ describing the sweep-out cone in transversal components in Fig. 7. Note from the analytical solutions derived in subsection 2.2 that these two functions share the angular frequency value, this implies redundancy in the data source employed to determine the first and second coast natural frequencies required in $\Delta w$, which in turn enables the use of Eq. (34). The existing redundancy represents an advantage of the proposed method that
Table 1: Main simulation parameters

\begin{tabular}{lrr}
\hline \multicolumn{1}{c}{ Variable } & Value & Remarks \\
\hline Keplerian orbital elements & & \\
Geometric altitude & 400 & $\mathrm{~km}$ \\
Eccentricity & 0.0014 & - \\
Inclination & 51 & $\circ$ \\
Longitude of the ascending node & 0 & $\circ$ \\
Argument of periapsis & 0 & $\circ$ \\
True anomaly & 270 & $\mathrm{~kg}$ \\
\hline CubeSats & & $\mathrm{kg} \mathrm{m}$ \\
Mass per U CubeSat & 1.3 & $\mathrm{~kg} \mathrm{~m}$ \\
$I_{t}$ & $\% / \mathrm{s}$ \\
$I_{z}$ & $0.0056-0.0372^{\dagger}$ & $\%$ \\
$\omega_{x_{0}}$ & $0.0039-0.0079^{\dagger}$ & $\%$ \\
$\omega_{y_{0}}$ & 1.1 & $\mathrm{~cm}^{2}$ \\
$\omega_{z_{0}}$ & 1.3 & \\
Total thruster area & 1.6 & $\mathrm{mdps} / \mathrm{dgit}^{\dagger}$ \\
\hline Digital gyroscope readings & & $\mathrm{mdps} / \sqrt{H z}$ \\
Sensitivity & 95 & \\
Rate Noise Density (RND) & & \\
\hline
\end{tabular}

Miscellaneous

Date and Time for the simulation 2014/03/20 12:00:00 Near Vernal Equinox Gravity Model

Thermospheric density model

Solar Irradiance Data

Moment arm GGM05G $10^{\text {th }}$ Degree and Order JB2008 SORCE

0.02375

* Alluding an ISS CubeSat deployment

${ }^{\dagger}$ For the $1.5 \mathrm{U}$ and $3 \mathrm{U}$ CubeSat respectively

$¥$ Compensated for gyroscopic bias drift

$\S$ Four times higher than the typical value $[1,10]$

becomes relevant in a real scenario wherein angular rate readings for each axis may differ in quality even from the same source rate sensor. Similarly to the discussion about the first manoeuvre, the span of the coasting periods under analysis are greatly dependant on specific mission constraints. For example, test energy consumption or eclipse conditions may be delimiting factors of the allowed analysis interval. Nevertheless, relatively short intervals would be sufficient for resolving welldefined natural frequencies in CubeSats as observed in Fig. 7.

Six different values of thrust have been used for the characterisation test simulation (second manoeuvre), namely $0.05 \mu \mathrm{N}$, $0.1 \mu \mathrm{N}, 0.5 \mu \mathrm{N}, 1 \mu \mathrm{N}, 5 \mu \mathrm{N}$, and $10 \mu \mathrm{N}$. These values correspond to the thrust delivered by each of the patch elements acting during the characterisation manoeuvre, specifically 1 and 4 in Fig. 3, adding spin about the $z$ body axis. A standard thrust characterisation based on $\omega_{z}$ has been used as figure of merit in the following descriptions. In all instances, the raw data has been conditioned using a Savitzky-Golay digital filter ${ }^{3}$. Note from Fig. 7 that the standard and proposed characterisation methods differ solely in the source component of $\boldsymbol{\omega}$ used in the computations. In the standard characterisation, the thrust magnitude is estimated directly from the temporal rate of change of $\omega_{z}$, namely angular acceleration $\alpha$, during the thrust campaign as illustrated in Fig. 7. In order to compute $\alpha$, a regression line has been fitted in the least-squares sense to the $\omega_{z}$ values ${ }^{4}$. On the other hand, $\omega_{y}$ has been picked to obtain $\Delta w$ from the coast-

\footnotetext{
${ }^{3}$ Savitzky-Golay FIR smoothing filter with polynomial order 1 and frame length $(s l)$ of 1001 samples. Smoothed values within the frame length are given by $\hat{\boldsymbol{\omega}}=\boldsymbol{B} \boldsymbol{\omega}$, with the smoothing filter matrix $\boldsymbol{B}=\boldsymbol{S}\left(\boldsymbol{S}^{T} \boldsymbol{S}\right)^{-1} \boldsymbol{S}^{T}$ where $\boldsymbol{S}_{i, j}=$ $\boldsymbol{Z}_{i}^{j-1}$ such that $(1-s l) / 2 \leq \boldsymbol{Z} \leq(s l-1) / 2$ and $s l$ is an odd number [37,pp. 427]

${ }^{4}$ If $\boldsymbol{\omega}_{z}$ and $\boldsymbol{t}$ are the analysed rate and time data vectors for respectively, then
} 


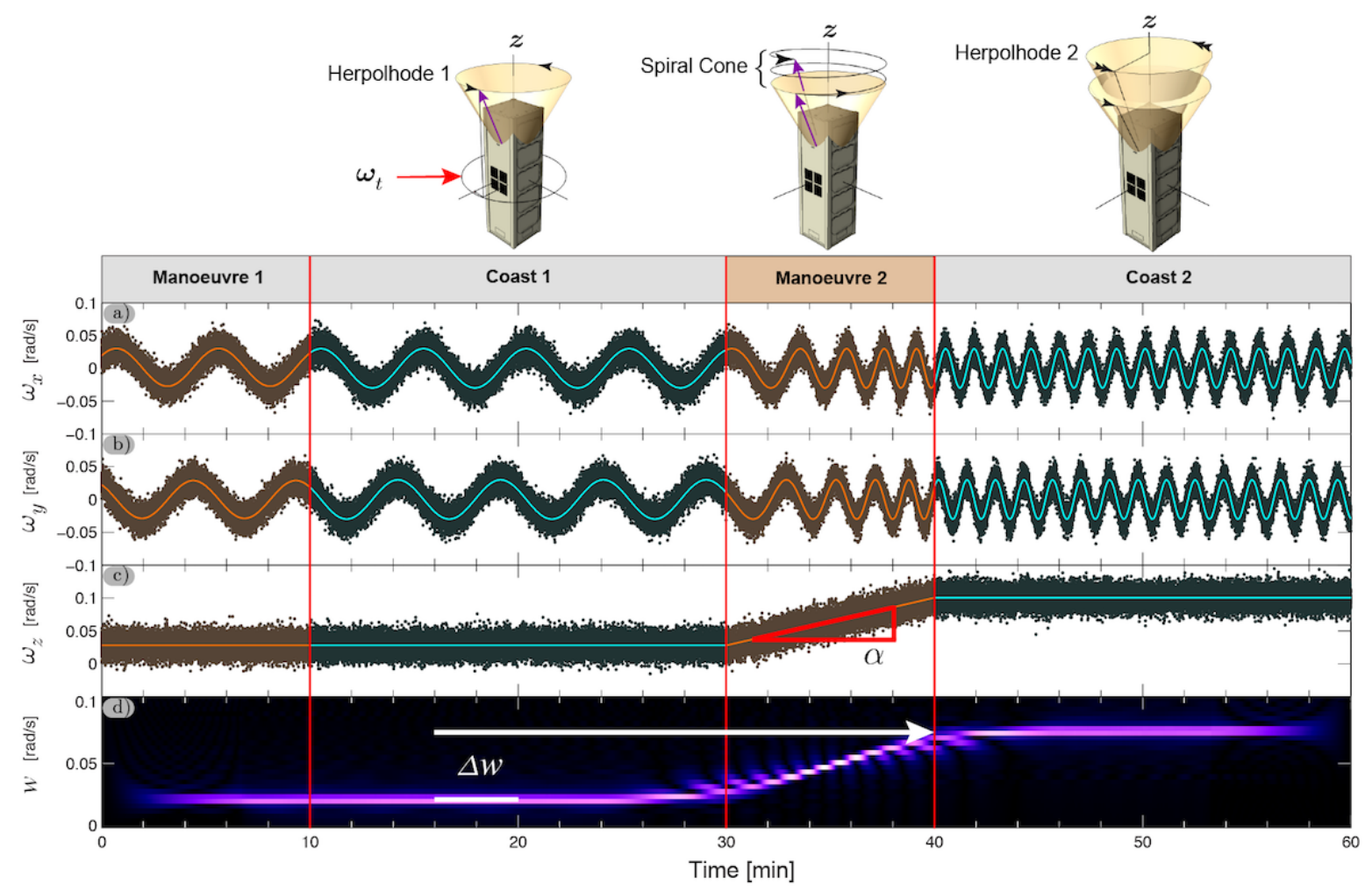

Figure 7: Example of angular velocities response. Plots a), b), and c) show noisy angular velocity signal outputs from the emulated digital gyro. Plot d) presents the Wigner-Ville spectrogram of $\omega_{y}$ evidencing a linear up-chirp signal along the manoeuvring time. Signal spectrum analysis is used in the example to identify component frequencies in the data.

ing periods 1 and 2. To this end, a Fast Fourier Transform has been used to identify the signal frequency of maximum energy that is subsequently used as initial guess in a fitting procedure aiming the refinement of the estimation. Finally, the provided spectrogram of $w$ in Fig. 7 illustrates the frequency evolution of $\omega_{y}$. Despite the apparent similarity between the plot of $\omega_{z}$ and the spectrogram of $w$, it is convenient to bear in mind that the first describes a magnitude whilst the latter describes the sweep-out frequency of the body cone.

Fig. 8 and Fig. 9 present the simulation results for a $1.5 \mathrm{U}$ and $3 \mathrm{U}$ CubeSat respectively. The statistical analysis comprises 20 samples for each of the 6 thrust levels for a total of 120 simulation samples for each CubeSat. The error percentage has been computed with respect to the nominal thrust value. Amplitude changes are highly susceptible to noise effects mostly when these approximate. In that case, thrust estimations from $\alpha$ are better represented from instantaneous changes during the manoeuvre instead that from pre and post manoeuvre data. Conversely, the intrinsic ease of identifying frequency changes rather than amplitude ones in rate noisy signals, confers the proposed method with the ability to utilise bigger portions of data. In this way, the observed consistent improved relative performance is clearly foreseen, considering that estimations from $\Delta w$ encompass four times the time span than those from $\alpha$ as

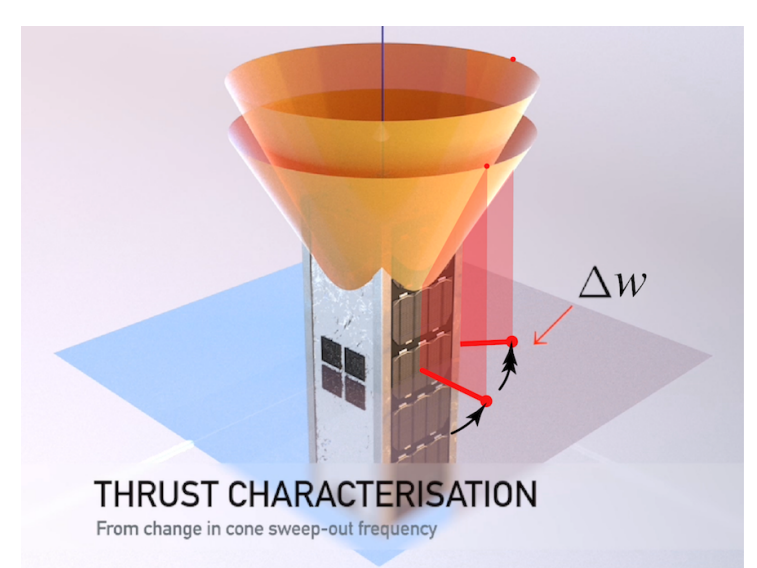

Video 1: Supplementary to Fig. 7, the video shows the body angular velocity response to thrust action and the proposed thrust characterisation method (online version only). The preview identifies the transversal natural frequency difference, that is the established difference between the baseline herpolhode sweep-out frequency during the Coast 1 and Coast 2 intervals. 


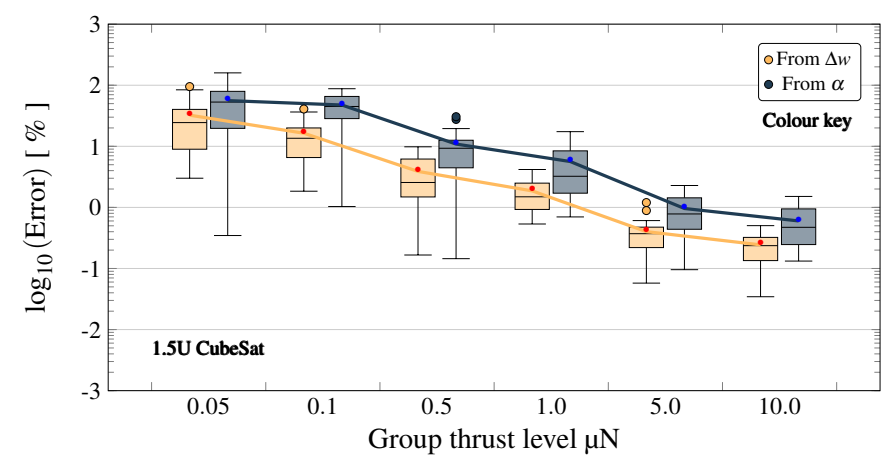

Figure 8: Log box-and-whisker plot of the error percentage with respect to the actual thrust level. Using estimations from the angular acceleration in $z$ body axis, $\alpha$, and from the difference in pre/post manoeuvre transversal natural frequencies, $\Delta w$, in a $1.5 \mathrm{U}$ CubeSat. Each box plot pair constitute a thrust test instance.

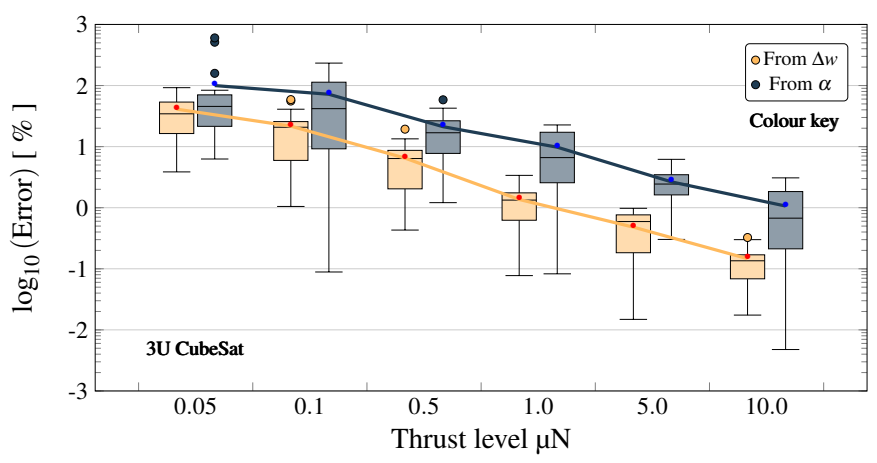

Figure 9: Log box-and-whisker plot of the error percentage with respect to the actual thrust level. Using estimations from the angular acceleration in $z$ body axis, $\alpha$, and from the difference in pre/post manoeuvre transversal natural frequencies, $\Delta w$, in a $3 \mathrm{U}$ CubeSat. Each box plot pair constitute a thrust test instance.

shown in Fig. 7. This important feature of the proposed method enables enhanced thrust estimations.

As a final remark in this section on the applicability of the proposed method, note from plots a) or b) in Fig. 7 that the angular sweep-out frequency has necessarily an upper limit before the appearance of frequency ambiguity in noisy signals. That limit is clearly defined by the quality of the signal under analysis, e.g. sensor properties.

\section{Conclusions}

This investigation set out to evaluate an alternative method to enhance in-orbit micro thrust characterisation using standard attitude determination elements in CubeSats.

Simulation results presented in this investigation show that it is possible to improve estimations of low thrust levels from weak and noisy sensor signals by evaluating the angular frequency of the transversal component of the angular velocity vector in intermediate axis spinners, over estimations from angular acceleration. Additionally, the proposed method adds versatility to the thrust analysis process by allowing selectable angular velocity transversal component.
This work contributes to existing knowledge of in-orbit thrust characterisation by providing a simple, accessible, robust and reliable method particularly valuable to CubeSat missions using simple COTS attitude determination components.

Although the proposed analysis is based on angular frequency changes rather than angular speeds, further research should focus on determining the effect, if any, of gyroscope bias drift on the performance of the method.

\section{Acknowledgments}

We would like to thank Nicholas H Crisp and Mostafa Nabawy for their valuable suggestions for improving this paper.

\section{Appendix}

\section{See Table 2}

\section{References}

[1] Analog (2006). ADIS16251 Programmable Low Power Gyroscope. Analog Devices Inc., Norwood, MA, USA, PrA edition.

[2] Arestie, S., Lightsey, E. G., and Hudson, B. (2012). Development of a modular, cold gas propulsion system for small satellite applications. Journal of Small Satellites, 1(2):63-74.

[3] Auman, A. J. (2015). Geometric attitude estimation \& orbit modelling. $\mathrm{PhD}$ thesis, University of Surrey.

[4] Barranger, J. (1965). Hysteresis and eddy-current losses of a transformer lamination viewed as an application of the pointing theorem. Washington: NASA Technical note.

[5] Boccaletto, L. and d'Agostino, L. (2000). Design and testing of a micronewton thrust stand for FEEP. In 36th AIAA/ASME/SAE/ASEE Joint Propulsion Conference, volume AIAA 2000-3268.

[6] Bulit, A., Gollor, M., Lionnet, P., Treuet, J.-C., and Gomez, I. A. (2015). D2.1 Database on EP (and EP-related) technologies and TRL. Technical report, EPIC-CNES.

[7] BUSEK (2017). ION thrusters BIT-3.

[8] Cal Poly (2015). CubeSat Design Specification (CDS) Rev 13. Cal Poly SLO, 2015 edition.

[9] ClydeSpace (2017). Pulsed plasma thrusters.

[10] CubeSatShop (2016). Nanopower solar P110U-A/B.

[11] Curtis, H. (2005). Orbital mechanics for engineering students, ch 1-5.

[12] DelfiSpace (2016). Delfi-n3xt micropropulsion payload.

[13] Dinelli, C., Samudra, H., Jin, H., Keidar, M., and Castonguay, K. (2014). Ballistic reinforced communication satellite (BRICSat-P): The first flight of an electric micropropulsion system for CubeSat mission applications. In The Interplanetary CubeSat Workshop, volume 2014.B.2.4. iCubeSat.

[14] eoPortal Directory (2017). Satellite missions database.

[15] FHWN (2017). Pegasus design.

[16] Frost, C., Agasid, E., et al. (2015). Small spacecraft technology state of the art. Moffett Field, California: NASA Ames Research Center.

[17] Gamero-Castaño, M. (2003). A torsional balance for the characterization of micronewton thrusters. Review of Scientific Instruments, 74(10).

[18] Gander, W., Gander, M., and Kwok, F. (2014). Scientific Computing - An Introduction using Maple and MATLAB. Texts in Computational Science and Engineering. Springer International Publishing.

[19] Glennon, E. P., Gauthier, J. P., Choudhury, M., Parkinson, K., and Dempster, A. G. (2013). Project Biarri and the Namuru V3.2 Spaceborne GPS Receiver. In International Global Navigation Satellite Systems Society Symposium.

[20] Goldstein, H., Poole, C. P., and Safko, J. L. (2014). Classical Mechanics: Pearson New International Edition. Pearson Higher Ed.

[21] Grubišić, A. and Gabriel, S. (2010). Development of an indirect counterbalanced pendulum optical-lever thrust balance for micro-to millinewton thrust measurement. Measurement Science and Technology, 21(10):105101. 
Table 2: Reported historical and planned CubeSat missions equipped with propulsion systems.

\begin{tabular}{|c|c|c|c|c|}
\hline CubeSat Name & Launch Date & Size Factor & Propellant & Details \\
\hline AeroCube 7B\&C (AC 7B\&C, OCSD B\&C) & 2016 & $1.5 \mathrm{U}$ & Water & $T_{\max }=5 \mathrm{mN}$ and $I_{s p}=75 \mathrm{~s}$ \\
\hline AeroCube $8 \mathrm{~A} \& \mathrm{~B}(\mathrm{AC} 8 \mathrm{~A} \& \mathrm{~B}, \text { IMPACT A\&B})^{\star}$ & 20/05/2015 & $1.5 \mathrm{U}$ & Polyether ether ketone & $T=12.5 \mu \mathrm{N}$ ea. ${ }^{\dagger}$ and $I_{s p}=1200 \mathrm{~s}$ [43], Scalable ion-Electrospray Propulsion system SiEPro \\
\hline BRICSat-P (NO 83, OSCAR 83) & 20/05/2015 & $1.5 \mathrm{U}$ & Titanium cathode & $T=1 \mu \mathrm{N}$ and $I_{s p}=3000 \mathrm{~s}$ [13], Micro-Cathode Arc Thruster $\mu \mathrm{CAT} T^{*}$ \\
\hline BRICSat-2 & 2017 & $1.5 \mathrm{U}$ & Titanium cathode & $T=1 \mu \mathrm{N}$ and $I_{s p}=3000 \mathrm{~s}[13]$, Micro-Cathode Arc Thruster $\mu \mathrm{CAT}^{*}$ \\
\hline NJUST-2 & $25 / 09 / 2015$ & $2 \mathrm{U}$ & - & Cold gas? [14] \\
\hline RAMPART $^{\star}$ & 2013 & $2 \mathrm{U}$ & DuPont Dymel-134a & $T=0.5 \mathrm{~N}$ and $I_{s p}=67-90 \mathrm{~s}$ [33], Warm-gas Resistojet \\
\hline Shankeda-2 & 25/09/2015 & $2 \mathrm{U}$ & - & Cold gas? [14] \\
\hline Pegasus (QB50 AT03) & 2017 & $2 \mathrm{U}$ & - & PPT [15] \\
\hline TW-1 (A/B/C Constellation) & $25 / 09 / 2015$ & $2-3 \mathrm{U}$ & Butane & $T=1 \mathrm{mN}, I=40 \mathrm{Ns}[44]$, Nanospace \\
\hline Biarri-Squad $1 \& 2 \& 3$ & 2016 & $3 \mathrm{U}$ & - & Microthrusters [19] \\
\hline URSA MAIOR (QB50 IT02) & 2017 & $3 \mathrm{U}$ & Nitrogen & $T=1 \mathrm{mN}[39]$ \\
\hline $\mathrm{CanX}-2^{\star}$ & $28 / 04 / 2008$ & $3 \mathrm{U}$ & $S F_{6}$ & $T=50 \mathrm{mN}$ and $I_{s p}=35-40 \mathrm{~s}$ [41], NanoPS \\
\hline CPOD A\&B & $01 / 01 / 2016$ & $3 \mathrm{U}$ & $\mathrm{R}-134 \mathrm{a}$ & 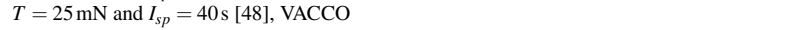 \\
\hline Delfi-n3Xt ${ }^{\star}$ & $21 / 11 / 2013$ & $3 \mathrm{U}$ & Nitrogen & $T=6-150 \mathrm{mN}[34]$ and $I_{s p}>30 \mathrm{~s}[12]$ \\
\hline${\mathrm{BEVO}-2^{\star}}^{\star}$ & 29/01/2016 & $3 \mathrm{U}$ & DuPont 236-fa & $T=110-150 \mathrm{mN}$ and $I_{s p}=65.7-89.5 \mathrm{~s}[2]$ \\
\hline LituanicaSAT 2 (QB50 LT01) & 2016 & $3 \mathrm{U}$ & LMP103S & $T=0.3 \mathrm{~N}$ and $\Delta v=200 \mathrm{~m} \mathrm{~s}^{-1}[35]$ \\
\hline NJFA-1 & $25 / 09 / 2015$ & $3 \mathrm{U}$ & - & Cold gas? [14] \\
\hline PATRIOT & - & $3 \mathrm{U}$ & - & $T=2 \mathrm{mN}$ and $I_{s p}=2000 \mathrm{~s}$ at an input power of $10 \mathrm{~W}$ [47], Ambipolar Thruster (CAT) \\
\hline POPSAT-HIP 1 & 19/06/2014 & $3 \mathrm{U}$ & Argon & $T=0.1-10 \mathrm{mN}$ and $I_{s p}=50 \mathrm{~s}$, Cold Gas Thruster [32] \\
\hline ALL-STAR PropSat & - & $3 \mathrm{U}$ & R-134a & Cold Gas Thruster [23] \\
\hline SERPENS $^{\star}$ & 19/08/2015 & $3 \mathrm{U}$ & Teflon & $T=40 \mu \mathrm{N}$ and $I_{s p}=600 \mathrm{~s}$ at an input power of $2 \mathrm{~W}[9]$, PPTCUP $\$$ \\
\hline STRaND- $1^{\star}$ & $25 / 02 / 2013$ & $3 \mathrm{U}$ & $\begin{array}{c}\text { Elkonite } \\
\text { Water-isopropanol }\end{array}$ & $\begin{array}{l}T=0.9 \mu \mathrm{N} \text { and } I_{s p}=1340 \mathrm{~s}[29], \mathrm{PPT} \\
\text { Resistojet }^{\star \star}[45]\end{array}$ \\
\hline SAMSON & 2017 & $6 \mathrm{U}$ & Teflon & $T=40 \mu \mathrm{N}$ and $I_{s p}=600 \mathrm{~s}$ at an input power of $2 \mathrm{~W}[6]$, PPTCUP $\$$ \\
\hline ARAPAIMA & - & $6 \mathrm{U}$ & Hexafluoropropane & $T=100 \mathrm{mN}$ and $I_{s p}=47 \mathrm{~s}[24]$ \\
\hline Lunar-IceCube & 2018 & $6 \mathrm{U}$ & Solid iodine & $T=1.4 \mathrm{mN}$ and $I_{s p}=3500 \mathrm{~s}$ [7], Miniature electric RF ion engine BIT- $3^{\S}$ \\
\hline MarCO $1 \& 2$ & 2018 & $6 \mathrm{U}$ & R-236fa & $T=25 \mathrm{mN}[49], \mathrm{VACCO}$ \\
\hline $\begin{array}{l}\text { ^ Propulsion technology demonstration mission } \\
\text { Values for the reported original S-iEPS module } \\
\$ \text { Operates at } 1-50 \mathrm{~Hz} \text { generating } 1 \mathrm{mNs} / \text { pulse and } \\
\$ \text { Commercial propulsion unit } \\
\text { ॠ A butane resistojet was originally proposed wi } \\
\text { Data collected from stated main references, an }\end{array}$ & $I_{s p}=2000-30$ & 00 s [30] & $\begin{array}{l}75 ? \mathrm{~s}[29] \\
\text { al Directory }\end{array}$ & \\
\hline
\end{tabular}

[22] Hamann, R., Bouwmeester, J., and Brouwer, G. (2009). Delfi-C3 preliminary mission results. 23rd Annual AIAA/USU Conference on Small Satellites, $\operatorname{SSC} 09-\operatorname{IV}(7)$.

[23] Hardy, P. E. (2013). Orbital maneuver applications and capabilities of the ALL-STAR CubeSat propulsion system.

[24] Harris, K., McGarvey, M., Chang, H., Ryle, M., Ruscitti, T., Udrea, B., and Nayak, M. (2013). Application for RSO Automated Proximity Analysis and IMAging (ARAPAIMA): Development of a nanosat-based space situational awareness mission. In CubeSat Summer Workshop 2013. EmbryRiddle Aeronautical University.

[25] Jamison, A. J., Ketsdever, A. D., and Muntz, E. (2001). Accurate measurement of nano-newton thrust for micropropulsion system characterization. In 27th International Electric Propulsion Conference, Pasadena, California, USA, IEPC-01-236.

[26] Janson, S. and Hinkley, D. (2009). Spin dynamics of the pico satellite solar cell testbed spacecraft. Small Satellite Conference.

[27] Janson, S. W. and Welle, R. P. (2014). The NASA optical communication and sensor demonstration program: An update. Technical Report SSC14VI-1, The Aerospace Corporation.

[28] Keidar, M., Zhuang, T., Shashurin, A., Teel, G., Chiu, D., Lukas, J., Haque, S., and Brieda, L. (2014). Electric propulsion for small satellites. Plasma Physics and Controlled Fusion, 57(1):014005.

[29] Kenyon, S. and Bridges, C. (2011). STRAND-1: Use of a \$500 smarthphone as the central avionics of a nanosatellite. In 62nd International Astronautical Congress, volume IAC-11-B4.6B.8.

[30] Lukas, J., Teel, G., Hague, S., Shashurin, A., and Keidar, M. (2014). Thruster subsystem design for the ballistic reinforced communication satellite. In CubeSat Developers Workshop, volume SSC14-WK-18. The George Washington University.

[31] Manzoni, G. and Brama, Y. L. (2015). CubeSat micropropulsion characterisation in low earth orbit. In 29th Annual AIAA/USU Conference on Small Satellites, volume SSC15-IV-5. Microspace Rapid Pte Ltd.

[32] Microspace Srl (2017). The power of microspace.

[33] Moore, G. (2010). 3D printing and MEMS propulsion for the RAMPART $2 \mathrm{U}$ CubeSat. In 24th Annual AIAA/USU Conference on Small Satellites, volume SSC10-III-8.

[34] Muller, C., Perez, L., Zandbergen, B., Brouwer, G., Amini, R., Kajon, D., and Sanders, B. (2010). Implementation of the T3 $\mu$ PS in the Delfi-n3Xt satellite. Small Satellite Missions for Earth Observation.

[35] Nano-Avionics (2017). LituanicaSAT-2.
[36] Neilsen, T., Weston, C., Fish, C., and Bingham, B. (2014). Dice: Challenges of spinning CubeSat. In 37th Annual AAS Guidance and Control Conference, Breckenridge, $\mathrm{CO}$.

[37] Orfanidis, S. J. (2010). Introduction to signal processing. Prentice-Hall, Inc.

[38] Packan, D., Bonnet, J., and Rocca, S. (2007). Thrust Measurements with the ONERA Micronewton Balance. 30th International Electric Propulsion Conference, 118.

[39] Piergentili, F., Balucani, M., Crescenzi, R., Piattoni, J., Santoni, F., Betti, B., Nasuti, F., and Onofri, M. (2013). MEMS cold gas microthruster on Ursa Maior CubeSat, volume 9, pages 7137-7143. International Astronautical Federation, IAF.

[40] Reijneveld, J. and Choukroun, D. (2013). Attitude control system of the Delfi-n3Xt satellite. Progress in Flight Dynamics, GNC, and Avionics 6 (2013) 189-208.

[41] Sarda, K., Grant, C., Eagleson, S., Kekez, D. D., and Zee, R. E. (2008). Canadian advanced nanospace experiment 2: On-orbit experiences with a three-kilogram satellite. In 22nd Annual AIAA/USU Conference on Small Satellites, volume SSC08-II-5. Space Flight Laboratory, University of Toronto Institute for Aerospace Studies.

[42] Soni, J. and Roy, S. (2013). Design and characterization of a nano-newton resolution thrust stand. Review of Scientific Instruments, 84(9):095103.

[43] SPL (2016). MIT SPL delivers the scalable ion electrospray propulsion system (S-iEPS) for CubeSats to NASA.

[44] SSCSpace (2017). Micropropulsion for nanosatellites.

[45] SSTL (2017). WARP DRiVE.

[46] Tsiotras, P. and Longuski, J. M. (1996). Analytic solution of Euler's equations of motion for an asymmetric rigid body. Journal of applied mechanics, 63(1):149-155.

[47] University of Michigan (2017). PEPL Thrusters: CubeSat Ambipolar Thruster.

[48] VACCO (2014). Reaction Control Propulsion Module X13003000-01. VACCO Industries, 7/14 edition.

[49] VACCO (2017). JPL MarCO micro propulsion system.

[50] Vadim, Y. (2016). CubeSat separation dynamics. In II Latin American IAA CubeSat WorkShop. 